\title{
Transcription factor cyclic adenosine monophosphate responsive element binding protein negatively regulates tumor necrosis factor alpha-induced protein 1 expression
}

\author{
NING LIU $^{1 *}$, KE WEI $^{1 *}$, YU XUN $^{1}$, XIAOXU YANG ${ }^{1}$, SHIQUAN GAN ${ }^{1}$, HUI XIAO ${ }^{1}$, YE XIAO ${ }^{1}$, FENG YAN ${ }^{1}$, \\ GUIE XIE $^{1}$, TINGTING WANG ${ }^{1}$, YINKE YANG ${ }^{2}$, JIAN ZHANG ${ }^{1}$, XIANG HU ${ }^{1}$ and SHUANGLIN XIANG ${ }^{1}$ \\ ${ }^{1}$ Key Laboratory of Protein Chemistry and Developmental Biology of the Education Ministry of China, \\ College of Life Science, Hunan Normal University; ${ }^{2}$ Department of Molecular Medicine, \\ College of Biology, Hunan University, Changsha, Hunan 410081, P.R. China
}

Received November 30, 2014; Accepted August 17, 2015

DOI: $10.3892 / \mathrm{mmr} .2015 .4336$

\begin{abstract}
Tumor necrosis factor alpha (TNF $\alpha)$-induced protein 1 (TNFAIP1) was originally identified as a protein involved in DNA replication, DNA damage repair, apoptosis and the progression of certain diseases, such as Alzheimer's disease. In the present study, forskolin, a stimulant of cyclic adenosine monophosphate (cAMP), was found to significantly reduce human TNFAIP1 mRNA levels and TNFAIPI promoter activity in the SKNSH human neuroblastoma cell line as indicated by polymerase chain reaction analysis and a luciferase reporter assay. The association between transcription factor cAMP response element-binding protein (CREB) and TNFAIP1 was further investigated using loss- and gain of function-studies with western blot analysis and luciferase reporter assays. The CREB-specific inhibitor KG-501 significantly increased TNFAIP1 protein levels, while overexpression of wild-type CREB, but not CREB mutated at ser133a or its DNA-binding site, significantly decreased human TNFAIP1 protein levels and TNFAIP1 promoter activity in
\end{abstract}

Correspondence to: Professor Shuanglin Xiang or Professor Xiang $\mathrm{Hu}$, Key Laboratory of Protein Chemistry and Developmental Biology of Education Ministry of China, College of Life Science, Hunan Normal University, 38 Lushan Road, Changsha, Hunan 410081, P.R. China

E-mail: xshlin@hunnu.edu.cn

E-mail: huxiang@hunnu.edu.cn

${ }^{*}$ Contributed equally

Abbreviations: CREB, cyclic adenosine monophosphate responsive element binding protein; ChIP, chromatin immunoprecipitation; siRNA, small interfering RNA; WT, wild-type; Del, deletion; Cons, consensus

Key words: tumor necrosis factor alpha-induced protein 1, cyclic adenosine monophosphate responsive element binding protein, transcriptional regulation, human, gene
SKNSH cells. Furthermore, two CRE sites located at -285 and -425 bp of the human TNFAIP1 promoter were identified to be responsible for CREB-induced inhibition of human TNFAIPI promoter activity. Chromatin immunoprecipitation assays confirmed that CREB bound to the TNFAIPI promoter region harboring these two CRE sites. A further luciferase reporter assay demonstrated that CREB phosphorylation on ser133 was responsible for forskolin-induced inhibition of TNFAIPI expression. In conclusion, the present study suggested that CREB is a negative regulator of the TNFAIP1 gene.

\section{Introduction}

Tumor necrosis factor alpha $(\mathrm{TNF} \alpha)$-induced protein 1 (TNFAIP1) was originally identified as a gene whose expression can be induced by $\mathrm{TNF} \alpha$ in umbilical vein endothelial cells (1). TNFAIPl is a highly conserved single-copy gene (1), implying that TNFAIP1 protein has an important physiological role, which, however, has remained to be sufficiently elucidated. Previous studies by our group have demonstrated that TNFAIP1 interacts with proliferating cell nuclear antigen and the small p50 sub-unit of DNA polymerase $\delta$, implying that it may be involved in DNA synthesis and DNA repair $(2,3)$. Another group reported that Ras homolog gene family, member B (RhoB) induces apoptosis by interacting with TNFAIP1 via a c-Jun $\mathrm{N}$-terminal kinase-mediated signaling mechanism, suggesting that TNFAIP1 is involved in apoptosis (4). Furthermore, TNFAIP1 is an adaptor for cullin 3 to control RhoA degradation and regulate the structure of the RhoA-associated actin cytoskeleton (5). In addition, the transcription levels of TNFAIPI were robustly induced in the brain of a transgenic Caenorhabditis elegans model of Alzheimer's disease (AD), indicating that TNFAIP1 may also be involved in AD development (6). Therefore, inhibition of TNFAIP1 expression may be beneficial for neuronal cells under pathological conditions, for which the elucidation of the transcriptional regulation mechanisms of TNFAIP1 expression is required. Previous studies have shown that transcription factor Sp1 is capable of controlling basal TNFAIP1 expression by directly binding to the proximal promoter region of 
human TNFAIP1 (7). However, the transcriptional regulation mechanisms have largely remained elusive.

The present study aimed to determine the effects of neuroprotective agents with activity against AD on the expression of TNFAIP1 in SKNSH human neuroblastoma cells using reverse-transcription polymerase chain reaction (RT-qPCR) analysis and a luciferase reporter assay. As forskolin (8) and genistein (9) have been demonstrated to potently stimulate cyclic adenosine monophosphate (cAMP) response element-binding protein (CREB)-mediated transcription (8), the present study investigated the role of transcription factor CREB in the regulation of TNFAIP1. Further mechanistic studies were performed by examining the effects of KG-501, a specific CREB inhibitor (10), and overexpression of wild-type (WT) CREB or CREB mutated at ser133a (CREBs133a) or at its DNA-binding site (KCREB) on the expression of TNFAIP1 expression as well as on TNFAIPl promoter activity in SKNSH cells. Furthermore, the present study aimed to identify the corresponding CREB binding sites in the promoter region of TNFAIP1, which were verified using luciferase reporter and chromatin immunoprecipitation (ChIP) assays. The present study elucidated the regulatory mechanisms of TNFAIPI by CREB, which will be useful for future studies regarding the regulation of TNFAIPI and the development of TNFAIP1-targeted therapeutics of neuronal disease.

\section{Materials and methods}

Materials. Lipofectamine $\mathrm{T}^{\mathrm{TM}}$ 2000, Dulbecco's modified Eagle's medium (DMEM), glutamine, penicillin and streptomycin were purchased from Invitrogen Life Technologies, Inc., Carlsbad, CA, USA). Fetal bovine serum (FBS) and $0.25 \%$ trypsin-EDTA were purchased from Gibco-BRL (Invitrogen Life Technologies, Inc.). Chemical compounds including dimethyl sulfoxide (DMSO), forskolin, valproic acid, polydatin, genistein, KG-501 and mouse monoclonal anti- $\beta$-actin antibody, were purchased from Sigma-Aldrich (St. Louis, MO, USA). Rabbit polyclonal anti-TNFAIP1 antibody, was custom-made by Nanjing Chuanbo Bioch Co. Ltd (Nanjing, China) according to the protocol of a previous study (3). Rabbit polyclonal anti-CREB antibody was purchased from Cell Signaling Technology, Inc. (Beverly, MA, USA). Cytomegalovirus plasmid (pCMV)-Myc and the CREB dominant-negative vector set, including pCMV-CREB, pCMV-CREBs133a, containing a mutation of the phosphorylatable serine 133, and pCMV-KCREB, containing a mutation of the DNA binding domain, were purchased from Clontech Inc. (Mountainview, CA, USA). pGEM ${ }^{\circledR}-T$ Easy Vector System II, pGL3-Basic vector and the Dual-Luciferase ${ }^{\circledR}$ Reporter Assay System were purchased from Promega (Madison, WI, USA). LA Taq ${ }^{\mathrm{TM}}$ DNA Polymerase with GC buffers was purchased from Takara (Otsu, Japan). The real-time PCR primers for human TNFAIP1 and $\beta$-actin were purchased from Qiagen $\mathrm{GmbH}$ (Hilden, Germany).

Reporter plasmid construction. The TNFAIP1 promoter region was previously characterized by Liu et al (7). The proximal 5' region of human TNFAIPI promoter region spanning from nucleotide -1087 to nucleotide -139 (GenBank accession number, NM_021137) was amplified by PCR using the forward primer P-948F (5'-GGGGTACCCCACA CATAACTGGCACTCA-3') and the reverse primer P-948R (5'-AAGCTTTCTCAGCAGCTGGGTGGCCA-3') using the genomic DNA of SKNSH cells as a template, and ligated into the pGL3-basic vector in the KpnI/HindIII sites, denoted as P-948 (-1087/-139). PCR was performed using a thermocycler (Eppendorf Mastercycler ${ }^{\circledR}$ Nexus; Eppendorf, Hamburg, Germany) with the following conditions: Initial denaturation of DNA, $94^{\circ} \mathrm{C}$ for $10 \mathrm{~min}$; denaturation, 32 cycles of $95^{\circ} \mathrm{C}$ for $45 \mathrm{sec}$; annealing, $58^{\circ} \mathrm{C}$ for $30 \mathrm{sec}$; extension, $72^{\circ} \mathrm{C}$ for $40 \mathrm{sec}$; and final extension, $72^{\circ} \mathrm{C}$ for $5 \mathrm{~min}$. The sequence of the promoter region of CRE from -1087 to $-139 \mathrm{bp}$ was predicted by Jaspar (http://jaspar.genereg.net/). Deletion of the CRE1 and CRE2 sites located at -285 and -425 bp was performed using overlapping extension PCR, as described previously (11). In brief, in the first round, two PCRs were performed in parallel using P-948 (-1087/-139) as a template: One PCR was performed with the forward primer P-948F and a reverse primer containing a CRE1-site deletion $[\operatorname{Pr}(\mathrm{p}) \mathrm{CRE} 1 \mathrm{mR}$ (5'-GAAGGTAGTGTAGGTAAACAGGCT-3')] or a primer containing a CRE2-site deletion $[\operatorname{Pr}(\mathrm{p}) \mathrm{CRE} 2 \mathrm{mR}$ (5'-AGGTAG GCGGTCGGGGGACGCGGG-3')]; the other PCR was performed with a forward primer containing a CRE1-site mutation $[\operatorname{Pr}(\mathrm{p}) \mathrm{CRE} 1 \mathrm{mF}$ (5'-TTTACCTACACTACCTTC CTGACTC-3')] or a forward primer containing a CRE2-site mutation $[\operatorname{Pr}(\mathrm{p}) \mathrm{CRE} 2 \mathrm{mF}$ (5'-CCCCCGACCGCCTACCTG CCGGCCC-3')] and the reverse primer P-1152R. In the second round, an equimolar mixture of the two PCR products was used as template with $\mathrm{P}-1152 \mathrm{~F}$ and $\mathrm{P}-1152 \mathrm{R}$ primers. The final PCR products were then cloned into pGL3-Basic vector in the KpnI/HindIII sites, denoted as P-948 (CRE1del) or P-948 (CRE2del). The above-mentioned PCR amplifications were performed using the Advantage 2 PCR kit (cat no. 639207; Clontech Laboratories, Inc., Mountainview, CA, USA). All primers were purchased from Sheng-Gong Technologies (Shanghai, China), and the construct sequences were confirmed by sequencing performed by Sheng-Gong Technologies.

Cell culture and transfection. SKNSH cells were obtained from the American Type Culture Collection (Manassas, VA, USA) and routinely cultured in a humidified atmosphere of 5\% $\mathrm{CO}_{2}$ at $37^{\circ} \mathrm{C}$ in DMEM supplemented with $10 \%$ fetal bovine serum, $0.3 \mathrm{mM}$ glutamine and $50 \mathrm{U} / \mathrm{ml}$ penicillin/streptomycin. Transient transfections were performed in cells at $70 \%$ confluence using Lipofectamine ${ }^{\mathrm{TM}} 2000$ reagent according to the manufacturer's instructions. After transfection for $4 \mathrm{~h}$, the transfection medium was replaced with DMEM.

Luciferase reporter assay. The luciferase assay was performed as previously described (12). In brief, SKNSH cells were transiently transfected with DNA constructs as well as pRL-TK, which was used as control for transfection efficiency. Cells were incubated for $24 \mathrm{~h}$ after transfection and were treated with reagents, including DMSO, polydatin (10 $\mu \mathrm{M})$, valproic acid $(10 \mu \mathrm{M})$, forskolin $(10 \mu \mathrm{M})$ and genistein $(10 \mu \mathrm{M})$ for another $24 \mathrm{~h}$. The cells were finally harvested and lysed using $1 \mathrm{X}$ passive lysis buffer, and luciferase activity was assessed utilizing the Dual-Luciferase ${ }^{\circledR}$ Reporter Assay System according to the manufacturer's instructions on a Veritas ${ }^{\mathrm{TM}}$ Microplate Luminometer (Turner 

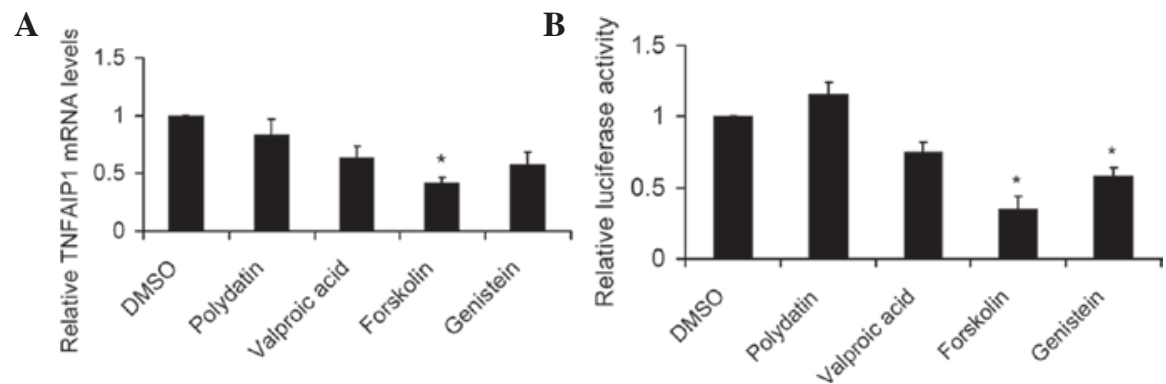

Figure 1. Forskolin inhibits TNFAIP1 mRNA levels and promoter activity. (A) The relative TNFAIP1 mRNA levels were examined by reverse-transcription polymerase chain reaction in SKNSH cells treated with DMSO, polydatin, valproic acid, forskolin or genistein. Values are expressed as the mean \pm standard deviation ( $\mathrm{n}=3$ ). (B) Relative luciferase activity of SKNSH cells transfected with P-948 luciferase reporter plasmid containing the (-1087/-139 bp) promoter region of TNFAIP1 was determined after treatment with DMSO, polydatin, valproic acid, forskolin or genistein for $24 \mathrm{~h}$. Values are expressed as the mean \pm standard deviation $(n=4)$. ${ }^{*} \mathrm{P}<0.05$ compared with cells treated with DMSO. DMSO, dimethyl sulfoxide.

BioSystems, Sunnyvale, CA, USA). Activity was defined as the Firefly/Renilla ratio.

RT-PCR analysis. After treatment with drugs including DMSO $(10 \mu \mathrm{M})$, forskolin $(10 \mu \mathrm{M})$, valproic acid $(10 \mu \mathrm{M})$, polydatin $(10 \mu \mathrm{M})$ or genistein $(10 \mu \mathrm{M})$ for $24 \mathrm{~h}$, the SKNSH cells were harvested for extraction of total RNA using TRIzol (Invitrogen Life Technologies, Inc.). Total mRNA was reversely transcribed into cDNA using the Superscript system (Invitrogen Life Technologies, Inc.). TNFAIP1 expression was analyzed by RT-PCR using a SYBR green kit (cat no. 4367659; Applied Biosystems Life Technologies, Foster City, CA, USA) according to the manufacturer's instructions on an ABI 7500 detection system (Applied Biosystems Life Technologies). The thermocycling conditions were as follows: Initial denaturation of DNA, $94^{\circ} \mathrm{C}$ for $5 \mathrm{~min}$; denaturation, 32 cycles of $95^{\circ} \mathrm{C}$ for $30 \mathrm{sec}$; annealing, $60^{\circ} \mathrm{C}$ for $30 \mathrm{sec}$; extension, $72^{\circ} \mathrm{C}$ for $30 \mathrm{sec}$; and final extension, $72^{\circ} \mathrm{C}$ for $5 \mathrm{~min}$. The RNA levels were determined using the $2^{-\Delta \Delta \mathrm{Ct}}$ method and expressed as the fold-change of TNFAIP1 expression in forskolin, valproic acid, polydatin or genistein-treated cells relative to DMSO-treated cells after normalization to the housekeeping gene $\beta$-actin.

Western blot analysis. Cell lysates were extracted from the SKNSH cells using M-PER Mammalian Protein Extraction Reagent (Cell Signaling Technology, Inc.) containing protease inhibitor (100X; Sigma-Aldrich, St. Louis, MO, USA), and the concentration of total proteins was determined by a Bicinchoninic Acid Protein assay kit (Pierce Biotechnology, Inc., Rockford, IL, USA). Protein $(20 \mu \mathrm{g})$ extracted from SKNSH cells was separated by $4-12 \%$ SDS-PAGE (Invitrogen Life Technologies, Inc.) and transferred onto a nitrocellulose membrane (EMD Millipore, Billerica, MA, USA) using the iBlot ${ }^{\circledR}$ dry blotting system (Invitrogen Life Technologies, Inc.). The membranes were blocked for $1 \mathrm{~h}$ at room temperature in $5 \%$ non-fat dried milk in $10 \mathrm{mM}$ phosphate-buffered saline (PBS; pH 7.2). Immunoblotting was then performed at $4^{\circ} \mathrm{C}$ overnight by incubation with the following primary antibodies: Rabbit polyclonal TNFAIP1 antibody (1:1,000; custom-made; Nanjing Chuanbo Biotech Co., Ltd., Nanjing, China), rabbit polyclonal CREB antibody (1:1,000; cat no. 4820; Cell Signaling Technology, Inc.) or mouse monoclonal $\beta$-actin antibody (1:5,000; cat no. A3854; Sigma-Aldrich). Following incubation with species-appropriate, horseradish peroxidase-conjugated secondary antibodies (1:5,000; cat no. M21001 for goat anti-mouse IgG-HRP; cat no. M21002 for goat anti-rabbit IgG-HRP; Abmart, Shanghai, China) for $1 \mathrm{~h}$ at room temperature, immunoreactive proteins were detected using enhanced chemiluminescence (ECL detection system; Amersham Pharmacia Biotech; GE Healthcare, Little Chalfont, UK) according to the manufacturer's instructions and then exposed to film (X-Omat; Eastman Kodak Co., Rochester, NY, USA). The intensity of the chemiluminescence signal was quantified by densitometry using ImageJ software version 1.49 (National Institutes of Health, Bethesda, MD, USA).

ChIP assay. ChIP assays were performed using the EZChIP ${ }^{\mathrm{TM}}$ kit (Upstate Biotechnology, EMD Millipore, Billerica, MA, USA) according to the manufacturer's instructions as previously described (12). In brief, $\sim 1 \times 10^{6}$ SKNSH cells were cross-linked with $1 \%$ formaldehyde and collected for sonication to shear the chromatin with an average size of 300 bp. Immunoprecipitation was then performed with anti-CREB antibody or control rabbit polyclonal immunoglobulin (Ig)G antibody. The DNA-protein complexes were then reverse cross-linked at $65^{\circ} \mathrm{C}$ for $3 \mathrm{~h}$. DNA from these samples was subjected to PCR with the following conditions: DNA initial denaturation, $94^{\circ} \mathrm{C}$ for $10 \mathrm{~min}$; denaturation, 32 cycles of $95^{\circ} \mathrm{C}$ for $50 \mathrm{sec}$; annealing, $58^{\circ} \mathrm{C}$ for $30 \mathrm{sec}$; extension, $72^{\circ} \mathrm{C}$ for $50 \mathrm{sec}$; and final extension, $72^{\circ} \mathrm{C}$ for $5 \mathrm{~min}$. Primer sets used for amplifying a 285-bp fragment spanning the 519-234 bp region of the TNFAIPl promoter were Ch-F (5'-GAATTC CCACGTCTCTCCCC-3') and Ch-R (5'-ATGGGGGCTGTA AGTGCTTC-3'). 18s ribosomal (r)RNA PCR was performed as a negative control. Primer sets used for amplifying a 233-bp DNA fragment corresponding to a region lacking CRE on the human 18s rRNA gene promoter were h18sF (5'-GTAACC CGTTGAACCCCATT-3') and h18sR (5'-CCATCCAATCGG TAGTAGCG-3'). The Agarose gel was prepared with $1.5 \%$ gel strength containing $1.0 \mu \mathrm{g} / \mathrm{ml}$ ethidium bromide (Sheng-Gong Technologies). PCR products were subjected to agarose gel electrophoresis (30 V for $1 \mathrm{~h}$ ) using the gel electrophoresis module (Beijing Liuyi Instrument Factory, Beijing, China).

Statistical analysis. Values are expressed as the mean \pm standard deviation of at least three independent experiments. Statistical analysis was performed using SPSS 18.0 software 
$\mathbf{A}$
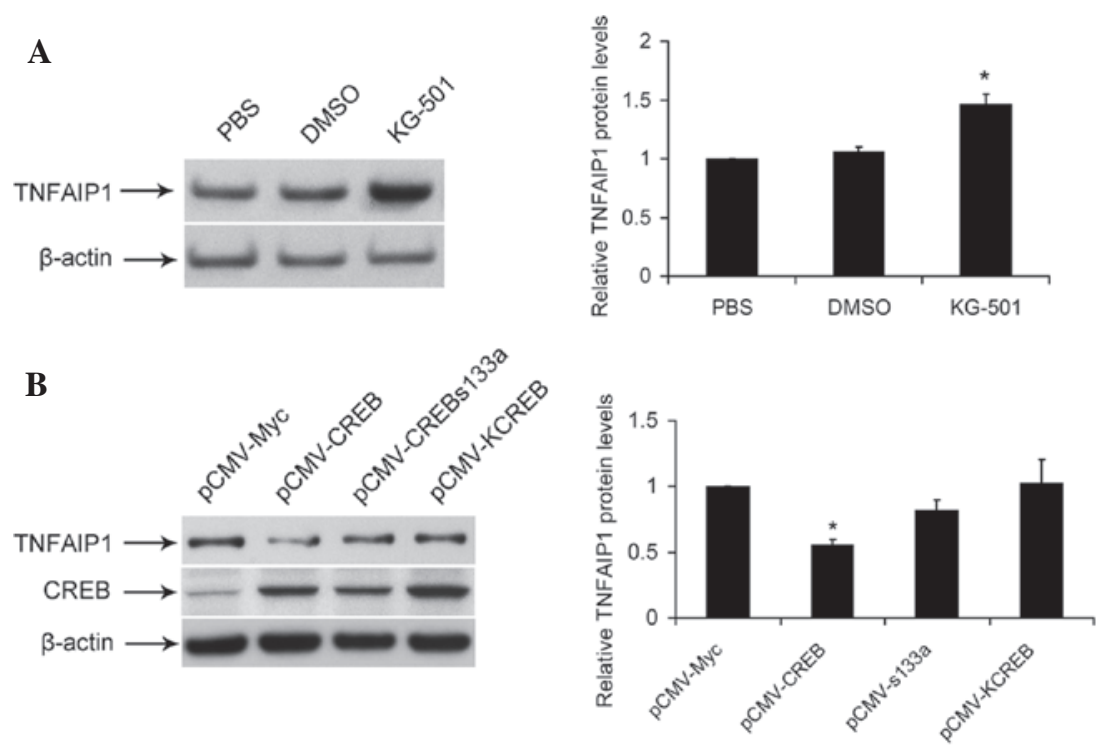

C

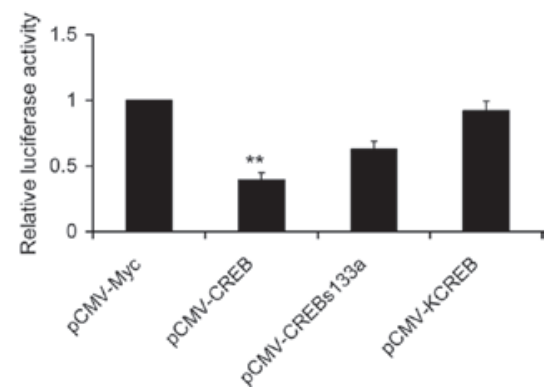

Figure 2. CREB negatively regulates TNFAIP1 expression and promoter activity. (A) TNFAIP1 protein expression in SKNSH cells after incubation with the specific CREB inhibitor KG-501 was detected by western blot analysis. $\beta$-actin was used as a loading control. Quantitative analysis of TNFAIP1 protein levels (fold of DMSO-treated cells; mean \pm standard deviation; $n=3$; ${ }^{*} \mathrm{P}<0.05$ compared with cells treated with DMSO). (B) TNFAIP1 protein expression in SKNSH cells after transfection with pCMV-Myc, wild-type or dominant-negative CREB vector was detected by western blot analysis. $\beta$-actin served as an equal loading control. Quantitative analysis of TNFAIP1 protein levels (fold of empty vector-transfected cells; mean \pm standard deviation; $n=3 ;{ }^{*} \mathrm{P}<0.05$ compared with cells transfected with pCMV-Myc vector). (C) Relative luciferase activity was determined after co-transfection of the P-948 luciferase reporter plasmid containing the (-1087/-139 bp) promoter region of TNFAIP1 with CREB and its dominant-negative mutant. Values are expressed as the mean \pm standard deviation $(\mathrm{n}=4) .{ }^{* *} \mathrm{P}<0.01$ compared with cells transfected with $\mathrm{pCMV}$-Myc vector. DMSO, dimethyl sulfoxide; PBS, phosphate-buffered saline; pCMV, cytomegalovirus plasmid; TNFAIP1; tumor necrosis factor alpha-induced protein 1; CREB, cyclic adenosine monophosphate responsive element binding protein.

(International Business Machines, Armonk, NY, USA). Results were analyzed by either Student's t-test or one-way analysis of variance. $\mathrm{P}<0.05$ was considered to indicate a statistically significant difference between values.

\section{Results}

Forskolin inhibits mRNA expression of TNFAIPI and TNFAIPI promoter activity in SKNSH cells. As TNFAIP1 was indicated to be involved in neuronal damage associated with $\mathrm{AD}$, the present study hypothesized that neuroprotective agents with activity against AD may target TNFAIP1. A group of chemical compounds, including valproic acid (13), polydatin (14), forskolin (15) and genistein (16) have been reported to exhibit therapeutic potential in AD. The effects of these chemical compounds on human TNFAIPI transcription and promoter activity in SKNSH cells were thus determined by RT-PCR and luciferase assay, respectively. The results showed that forskolin significantly inhibited human TNFAIPI mRNA levels in SKNSH cells (Fig. 1A). Similarly, forskolin and genistein significantly attenuated the activity of the P-948 (-1087/-139) TNFAIP1 promoter (Fig. 1B). Forskolin is a selective activator of adenylate cyclase that potently increases phosphorylation of CREB on its ser-133 site to stimulate CREB-mediated transcription (17); thus, CREB may be involved in the regulation of TNFAIP1 expression by forskolin.

CREB acts as a negative transcriptional regulator of TNFAIPI expression. To examine the roles of CREB in TNFAIP1 regulation, the present study used CREB inhibitor KG-501. The results showed that KG-501 significantly increased the protein expression of TNFAIP1 in SKNSH cells (Fig. 2A). Next, the present study examined the effects of CREB and dominant-mutant CREB vectors (KCREB and CREBs133a) on the protein expression of TNFAIP1. As expected, overexpression of CREB resulted in a decrease in TNFAIP1 protein expression, while KCREB or CREBs133a had no significant effect (Fig. 2B). Furthermore, the present study evaluated the effects of CREB on TNFAIP1 promoter activity. SKNSH cells were transiently transfected with P-948 (-1087/-139) and WT CREB expression vector or dominant-mutant CREB vectors (KCREB and CREBs133a). WT CREB, but not the dominant-mutant CREB vectors significantly reduced TNFAIP1 promoter activity (Fig. 2C). In conclusion, these results suggested that CREB negatively regulates TNFAIPI expression.

CREB suppresses TNFAIPI promoter activity through CREs. As CREB repressed TNFAIPI promoter activity, the present study further analyzed the potential CREs that are able to bind to CREB at sites located in the region of -1087 to -139 by using the bioinformatics transcription factor binding site prediction tool Jaspar (http://jaspar.genereg.net/). Two putative cAMP response elements, designated CRE1 and CRE2, were discovered at -285 and $-425 \mathrm{bp}$, respectively (Fig. 3A). To investigate whether these CRE sites mediate CREB-decreased TNFAIPI promoter activity, deleting mutations of these two CRE sites were generated (Fig. 3B). SKNSH cells were co-transfected with CREB expression vector or empty vector as well as P-948 (-1087/-139), P-984 (CRE1del) or P-984 (CRE2del). A luciferase assay showed that CREB overexpression significantly 
A

$-1087$

CCACACATAACTGGCACTCAGTAAATGTTTGTGGAATGAATGACCGTGGAAACAGG Forward primer

AGACTATTGCAGTAACCAAGGCTGGCAGCCGAACACAAGTGTTCCGAGTTGAGATGT TTTGTAAGTCGAGGTGATGGGGCTTAGCAATGGACTGACTGAATGTGGGGGTTTGGG GATAAGAGAAAAACAAGTCAGGCATGACTCCCATGAATCTGGCTTGGCCCACCTATC TGATTGGAAGGGCTGGCTTAAAGAGATAGGTAAGATGGAAGAAGAGGTGCAGGGAA AGAGTTCACAGATTATCTACCAACTCCCATCACACGCGTGTCTGAGCATCTGAGCAA AGAOTCACAGATACTCACT 作 AAACTCCCCGAACTTATCCGAGCCGCAGTTTCCTCGTAAGATCGTGGCAAGCATCAA CGCATCAAACTCCAGGCCTAAGCTGGGTGTGAAAGCGCCCCCCTGAGAGTGGGGCG GAATTCCCACGTCTCTCCCCGCCCGGTACTGCCTTTCCTCAGGACGCGGGCCTGTCAG GCTCCTCTCGCCCCTGGTCCGCCCGCGTCCCCCGACCCGAGGTCAGCCTACCTGCCG CRE2 site (-425)

GCCCCGCGGCGGCAGCGCGCTGCAGCCCAGGCTGATGTAGACAGGCAGCTTCTCCTG GGTCAGCGACAGCCGGTACCCAGCCAGCCTGCCACGGCCGCTGCCACGGATACAGA CRE1 site (-285)

TTCCACAGAGCCTGCTGGGAAACAGAGGCCCGAGGGGAGGCCCGGCCTACAAGACC

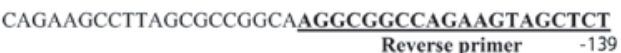

B

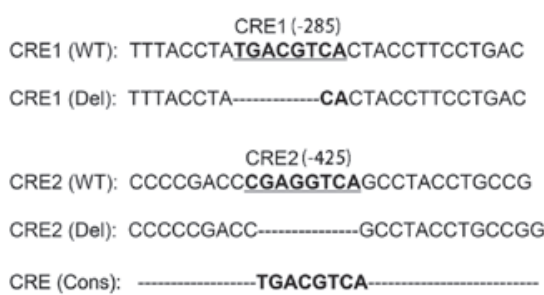

C

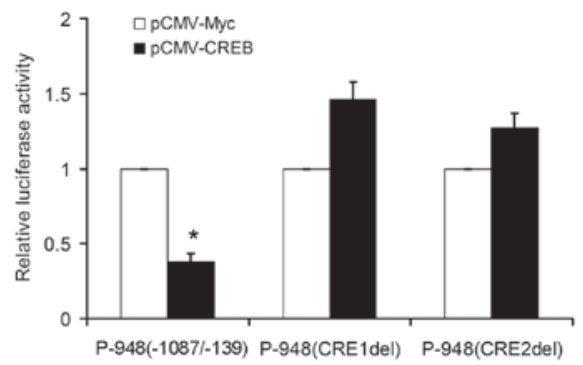

Figure 3. CRE1 and CRE2 are responsible for CREB-induced inhibition of TNFAIP1 promoter activity. (A) Bioinformatic analysis of the human TNFAIP1 promoter region spanning from $-1,087$ to -139 using the JASPAR database (http://jaspar.genereg.net/). Two putative CRE sites are indicated. (B) Deleting mutations in the putative CRE1 and CRE2 sites. Bold letters indicate putative CRE1, CRE2 sites and the CRE consensus sequence. (C) Relative luciferase activity (mean \pm standard deviation; $n=4$ ) was determined at $24 \mathrm{~h}$ after co-transfection of pCMV-myc or pCMV-CREB with P-948 (-1087/-139) or P-984 (CRE1del) or P-984 (CRE2del). * $\mathrm{P}<0.05$ compared with cells transfected with pCMV-Myc and P-948 (-1087/-139). pCMV, cytomegalovirus plasmid; TNFAIP1; tumor necrosis factor alpha-induced protein 1; CREB, cyclic adenosine monophosphate responsive element binding protein; P-948 (-1087/-139), P-948 luciferase reporter plasmid containing the (-1087/-139 bp) promoter region of TNFAIP1; WT, wild-type; Del, deletion; Cons, consensus

decreased the promoter activity of P-948 (-1087/-139), while CREB overexpression did not cause inhibition of the promoter activity of P-948 (CRE1del) and P-948 (CRE2del; Fig. 3C). These results indicate that CRE1 and CRE2 are involved in the suppression of TNFAIP1 expression by CREB.

CREB is capable of binding to the TNFAIP1 promoter. The present study further investigated whether CREB suppresses TNFAIPl gene expression through directly binding to the TNFAIP1 promoter. To test this, ChIP assays were performed with CREB antibody or IgG controls in SKNSH cells. DNA precipitated by ChIP was amplified by PCR using TNFAIPI

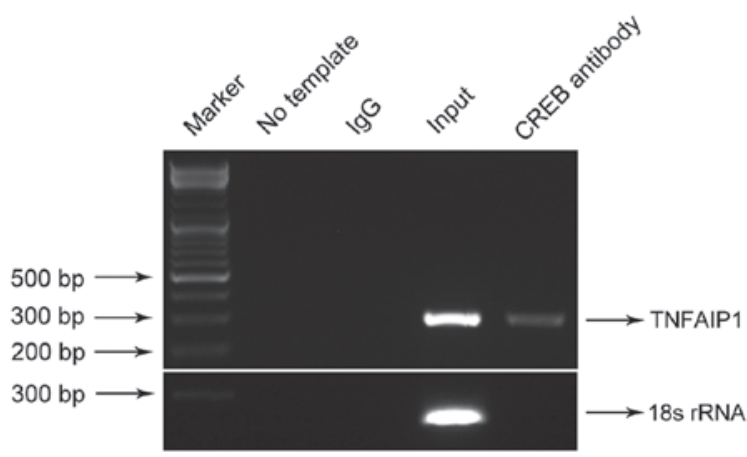

Figure 4. CREB binds to the human TNFAIP1 promoter region in SKNSH cells. Chromatin immunoprecipitation was performed in SKNSH cells to analyze the interactions of CREB with the human TNFAIP1 promoter covering two CREs or the human $18 s$ rRNA promoter lacking a CRE. Rabbit IgG was used as the negative control. IgG, immunoglobulin G; CREB, cyclic adenosine monophosphate responsive element binding protein; rRNA, ribosomal RNA.

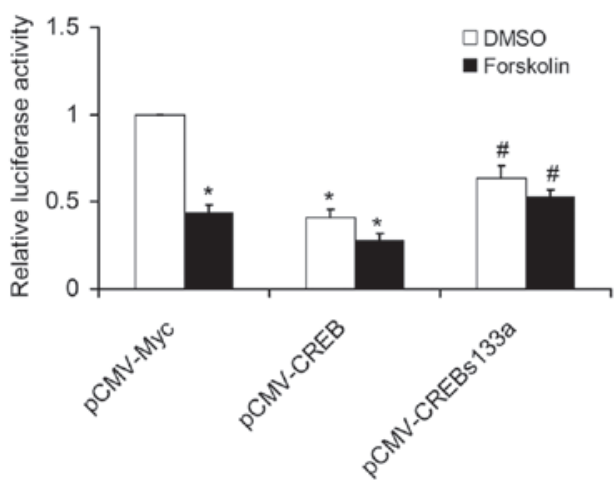

Figure 5. Phosphorylation of CREB on ser133 is necessary for forskolin-induced inhibition of human TNFAIP1 promoter activity. Relative luciferase activity was determined after co-transfection of P-948 luciferase reporter plasmid containing the (-1087/-139 bp) promoter region of TNFAIP1 with CREB or its dominant-negative mutant for $24 \mathrm{~h}$, followed by treatment with DMSO or forskolin for another $24 \mathrm{~h}$. Values are expressed as the mean \pm standard deviation $(n=3)$. ${ }^{*} \mathrm{P}<0.05$ compared with $\mathrm{pCMV}-\mathrm{Myc}$ vector-transfected cells treated with DMSO. ${ }^{\text {P }} \mathrm{P}<0.05$ compared with pCMV-CREB vector-transfected cells. CREB, cyclic adenosine monophosphate responsive element binding protein; $\mathrm{PCMV}$, cytomegalovirus plasmid; DMSO, dimethyl sulfoxide.

promoter-specific primers covering the two CRE sites from -519 bp to -234 bp or 18 s rRNA promoter primers lacking CRE sites. Analysis of the PCR products by gel electrophoresis showed that CREB was able to bind to the promoter region of TNFAIP1 covering two CREs, but not to the 18 s rRNA lacking CRE sites (Fig. 4). ChIP PCR therefore confirmed that CREB binds to the TNFAIP1 promoter region in SKNSH cells.

Forskolin decreases TNFAIP1 expression mediated by CREB phosphorylation on ser133. The results of the present study suggested that CREB is a negative regulator for TNFAIP1 expression. Therefore, it was further investigated whether CREB is involved in forskolin-induced inhibition of TNFAIPI expression. In accordance with the results shown in Figs. 1B and $2 \mathrm{C}$, treatment with forskolin or CREB overexpression significantly decreased TNFAIPI promoter activity, and forskolin combined with CREB overexpression further reduced TNFAIP1 promoter activity (Fig. 5). However, although an 
identical dose of forskolin was used, TNFAIP1 promoter activity was partially restored in CREBs133a-transfected cells (Fig. 5). These results indicated that CREB phosphorylation on ser133 is responsible for forskolin-induced inhibition of TNFAIP1 expression.

\section{Discussion}

TNFAIP1 has been suggested to be an apoptosis-associated protein (4) and involved in the development of AD (6). Therefore, TNFAIP1 is likely to be implicated in neuronal damage associated with neurodegenerative disease. Indeed, this notion was confirmed by experiments performed in our group, which demonstrated that inhibition of TNFAIP1 expression decreased $A \beta$-induced neuronal toxicity (unpublished data). Therefore, inhibition of TNFAIPI expression under neuropathological conditions may have neuroprotective effects. Elucidation of the transcriptional regulation mechanisms of TNFAIP1 may aid in the development of therapeutics for neuronal disease targeting TNFAIP1. The present study was the first, to the best of our knowledge, to demonstrate that CREB is a critical transcription factor for the negative regulation of TNFAIPI transcription in SKNSH cells.

Given that TNFAIP1 is implicated in the development of $\mathrm{AD}$ and is induced in the generation of animal models of $\mathrm{AD}$, therapeutic drugs with activity against AD have been hypothesized to inhibit TNFAIP1 expression. In the present study, a number of well-known natural products, including polydatin, valproic acid, forskolin and genistein, were selected for the evaluation of their inhibitory effects on TNFAIP1 expression, as they have been demonstrated to be potentially protective against AD (13-16). The results showed that polydatin and valproic acid slightly inhibited TNFAIP1 promoter activity. Of note, forskolin and genistein significantly suppressed TNFAIP1 promoter activity. To date, no study has indicated that polydatin is able to activate CREB, whereas valproic acid (18), forskolin (8) and genistein (9) are known to potently stimulate the phosphorylation of CREB at ser133 and consequently activate CREB-mediated transcription (8). These findings indicated that transcription factor CREB may be a potential inhibitor of TNFAIP1 expression in neuronal cells. Genistein is a known phytoestrogen with marked structural and functional similarity with $17 \beta$-estradiol (19). A recent study suggested that $17 \beta$-estradiol is a negative regulator of TNFAIP1 in mouse hippocampi (20). These findings were consistent with the results of the present study, demonstrating that TNFAIP1 expression was negatively regulated by genistein.

CREB is a key transcription factor that is tightly linked with neuronal cell survival and apoptosis $(21,22)$. Upon stimulation, CREB is phosphorylated at ser133 and subsequent recruited to $\operatorname{CRE}(23,24)$. As expected, the present study revealed that overexpression of WT CREB, but not mutated CREB (CREBs133a and KCREB) significantly inhibited TNFAIP1 promoter activity as well as protein expression. In addition, the present study demonstrated that WT CREB promoted forskolin-induced inhibition of TNFAIPI promoter activity, which was, however, partially rescued by overexpression of CREBs133a. It has been suggested that dominant-negative CREB interfered with the function of the phosphorylated CREB, possibly via the formation of inactive heterodimers (25). Thus, based on the results of the present study, phosphorylation of CREB at ser133 is necessary for CREB-induced downregulation of TNFAIPI and activation of upstream signaling pathways leading to CREB phosphorylation may thus intervene with TNFAIPI expression.

As it was demonstrated that TNFAIPI promoter activity was inhibited by transcription factor CREB, the present study further analyzed the CRE sites in the TNFAIP1 promoter spanning from -1087 to $-139 \mathrm{bp}$. Two CRE sites, CRE1 and CRE2, were identified using bioinformatics analysis. In fact, a previous study indicated that site-directed mutagenesis of the CRE1 site had no effect on the basal TNFAIP1 promoter activity (7). However, the results of the present study showed that a deleting mutation of CRE1 and CRE2 inhibited the CREB-induced reduction of TNFAIPI promoter activity. It is possible that CREB binds to the CRE1 site upon stimulation, but not under basal conditions. Although the ChIP assay of the present study showed that CREB was able to bind to the TNFAIP1 promoter region from - 519 to $-234 \mathrm{bp}$ covering the two CRE sites, is not possible to conclude that CREB specifically binds to CRE1 or CRE2 sites. Further study is required to determine whether CREB specifically interacts with the CRE1 or the CRE2 site under basal and pathological conditions.

In conclusion, the present study suggested that forskolin suppressed the expression of pro-apoptotic protein TNFAIP1 through activating neuronal survival mediator CREB. Recent experiments performed in our group have shown that overexpression of TNFAIP1 in neuronal cells induces significant neuronal apoptosis (unpublished data). Therefore, the present study links neuronal survival mediator CREB and apoptosis-associated protein TNFAIP1, which is likely to be involved in the development of AD. The present study thus provided a molecular basis for the intervention of neuronal disorders by targeting TNFAIP1. Whether the expression of TNFAIP1 is associated with neuropathological conditions will be investigated in future studies.

\section{Acknowledgements}

The present study was supported in part by the Cooperative Innovation Center of Engineering and New Products for Developmental Biology of Hunan Province, the 973 Project of the Ministry of Science and Technology of China (no. 2010CB529900), the China Postdoctoral Science Foundation (no. 2014M552139), the Research Fund for the Doctoral Program of Higher Education of China (no. 20104306110005), the Hunan Postdoctoral Science Foundation (no. 2012RS4016) and the Science and Technology Project of the Provincial Department of Education (no. 2014RS4008).

\section{References}

1. Wolf FW, Marks RM, Sarma V, Byers MG, Katz RW, Shows TB and Dixit VM: Characterization of a novel tumor necrosis factor-alpha-induced endothelial primary response gene. J Biol Chem 267: 1317-1326, 1992.

2. Zhou J, Hu X, Xiong X, Liu X, Liu Y, Ren K, Jiang T, Hu X and Zhang J: Cloning of two rat PDIP1 related genes and their interactions with proliferating cell nuclear antigen. J Exp Zool A Comp Exp Biol 303: 227-240, 2005. 
3. Yang L, Liu N, Hu X, Zhang W, Wang T, Li H, Zhang B, Xiang S, Zhou J and Zhang J: CK2 phosphorylates TNFAIP1 to affect its subcellular localization and interaction with PCNA. Mol Biol Rep 37: 2967-2973, 2010

4. Kim DM, Chung KS, Choi SJ, Jung YJ, Park SK, Han GH, Ha JS, Song KB, Choi NS, Kim HM, et al: RhoB induces apoptosis via direct interaction with TNFAIP1 in HeLa cells. Int J Cancer 125: 2520-2527, 2009.

5. Chen Y, Yang Z, Meng M, Zhao Y, Dong N, Yan H, Liu L, Ding M, Peng HB and Shao F: Cullin mediates degradation of RhoA through evolutionarily conserved BTB adaptors to control actin cytoskeleton structure and cell movement. Mol Cell 35: 841-855, 2009.

6. Link CD, Taft A, Kapulkin V, Duke K, Kim S, Fei Q, Wood DE and Sahagan BG: Gene expression analysis in a transgenic Caenorhabditis elegans Alzheimer's disease model. Neurobiol Aging 24: 397-413, 2003.

7. Liu M, Sun Z, Zhou A, Li H, Yang L, Zhou C, Liu R, Hu X, Zhou J, Xiang S and Zhang J: Functional characterization of the promoter region of human TNFAIP1 gene. Mol Biol Rep 37: $1699-1705,2010$

8. Johannessen M, Delghandi MP, Seternes OM, Johansen B and Moens U: Synergistic activation of CREB-mediated transcription by forskolin and phorbol ester requires PKC and depends on the glutamine-rich Q2 transactivation domain. Cell Signal 16: 1187-1199, 2004.

9. Sárvári M, Szego EM, Barabás K, Jávor A, Tóth S, Kovács Z and Abrahám IM: Genistein induces phosphorylation of cAMP response element-binding protein in neonatal hypothalamus in vivo. J Neuroendocrinol 21: 1024-1028, 2009.

10. Best JL, Amezcua CA, Mayr B, Flechner L, Murawsky CM, Emerson B,Zor T, Gardner KH and Montminy M: Identification of small-molecule antagonists that inhibit an activator: Coactivator interaction. Proc Natl Acad Sci USA 101: 17622-17627, 2004

11. Liu N, Yu Z, Li Y, Yuan J, Zhang J, Xiang S and Wang X: Transcriptional regulation of mouse neuroglobin gene by cyclic AMP responsive element binding protein (CREB) in N2a cells Neurosci Lett 534: 333-337, 2013.

12. Liu N, Yu Z, Xiang S, Zhao S, Tjarnlund-Wolf A, Xing C, Zhang $\mathrm{J}$ and Wang $\mathrm{X}$ : Transcriptional regulation mechanisms of hypoxia-induced neuroglobin gene expression. Biochem J 443: $153-164,2012$.

13. Wang Z, Zhang XJ, Li T, Li J, Tang Y and Le W: Valproic acid reduces neuritic plaque formation and improves learning deficits in $\mathrm{APP}(\mathrm{Swe}) / \mathrm{PS} 1(\mathrm{~A} 246 \mathrm{E})$ transgenic mice via preventing the prenatal hypoxia-induced down-regulation of neprilysin. CNS Neurosci Ther 20: 209-217, 2014.
14. Li RP, Wang ZZ, Sun MX, Hou XL, Sun Y, Deng ZF and Xiao K: Polydatin protects learning and memory impairments in a rat model of vascular dementia. Phytomedicine 19: 677-681, 2012.

15. Vitolo OV, Sant'Angelo A, Costanzo V, Battaglia F, Arancio O and Shelanski M: Amyloid beta-peptide inhibition of the PKA/CREB pathway and long-term potentiation: Reversibility by drugs that enhance cAMP signaling. Proc Natl Acad Sci USA 99: 13217-13221, 2002.

16. Bagheri M, Joghataei MT, Mohseni S and Roghani M: Genistein ameliorates learning and memory deficits in amyloid $\beta(1-40)$ rat model of Alzheimer's disease. Neurobiol Learn Mem 95: 270-276, 2011.

17. Gonzalez GA and Montminy MR: Cyclic AMP stimulates somatostatin gene transcription by phosphorylation of CREB at serine 133. Cell 59: 675-680, 1989 .

18. Biermann J, Grieshaber P, Goebel U, Martin G, Thanos S, Di Giovanni S and Lagrèze WA: Valproic acid-mediated neuroprotection and regeneration in injured retinal ganglion cells. Invest Ophthalmol Vis Sci 51: 526-534, 2010.

19. Lovekamp-Swan T, Glendenning M and Schreihofer DA: A high soy diet reduces programmed cell death and enhances bcl-xL expression in experimental stroke. Neuroscience 148: 644-652, 2007.

20. Liu H, Yang L, Zhao Y, Zeng G, Wu Y, Chen Y, Zhang J and Zeng Q: Estrogen is a novel regulator of Tnfaip1 in mouse hippocampus. Int J Mol Med 34: 219-227, 2014.

21. Walton M, Woodgate AM, Muravlev A, Xu R, During MJ and Dragunow M: CREB phosphorylation promotes nerve cell survival. J Neurochem 73: 1836-1842, 1999.

22. Mabuchi T, Kitagawa K, Kuwabara K, Takasawa K, Ohtsuki T, Xia Z, Storm D, Yanagihara T, Hori M and Matsumoto M: Phosphorylation of cAMP response element-binding protein in hippocampal neurons as a protective response after exposure to glutamate in vitro and ischemia in vivo. J Neurosci 21: 9204-9213, 2001.

23. Chrivia JC, Kwok RP, Lamb N, Hagiwara M, Montminy MR and Goodman RH: Phosphorylated CREB binds specifically to the nuclear protein CBP. Nature 365: 855-859, 1993.

24. Parker D, Ferreri K, Nakajima T, LaMorte VJ, Evans R, Koerber SC, Hoeger C and Montminy MR: Phosphorylation of CREB at Ser-133 induces complex formation with CREB-binding protein via a direct mechanism. Mol Cell Biol 16: 694-703, 1996.

25. Wilson BE, Mochon E and Boxer LM: Induction of bcl-2 expression by phosphorylated CREB proteins during B-cell activation and rescue from apoptosis. Mol Cell Biol 16: 5546-5556, 1996. 